\title{
Stress response and structural transitions in sheared gyroidal and lamellar amphiphilic mesophases: lattice-Boltzmann simulations
}

\section{Citation for published version (APA):}

González-Segredo, N., Harting, J. D. R., Giupponi, G., \& Coveney, P. V. (2006). Stress response and structural transitions in sheared gyroidal and lamellar amphiphilic mesophases: lattice-Boltzmann simulations. Physical Review E - Statistical, Nonlinear, and Soft Matter Physics, 73(3), 031503-1/12. [031503].

https://doi.org/10.1103/PhysRevE.73.031503

DOI:

10.1103/PhysRevE.73.031503

Document status and date:

Published: 01/01/2006

Document Version:

Publisher's PDF, also known as Version of Record (includes final page, issue and volume numbers)

\section{Please check the document version of this publication:}

- A submitted manuscript is the version of the article upon submission and before peer-review. There can be important differences between the submitted version and the official published version of record. People interested in the research are advised to contact the author for the final version of the publication, or visit the DOI to the publisher's website.

- The final author version and the galley proof are versions of the publication after peer review.

- The final published version features the final layout of the paper including the volume, issue and page numbers.

Link to publication

\footnotetext{
General rights

- You may freely distribute the URL identifying the publication in the public portal. follow below link for the End User Agreement:

www.tue.nl/taverne

\section{Take down policy}

If you believe that this document breaches copyright please contact us at:

openaccess@tue.nl

providing details and we will investigate your claim.
}

Copyright and moral rights for the publications made accessible in the public portal are retained by the authors and/or other copyright owners and it is a condition of accessing publications that users recognise and abide by the legal requirements associated with these rights.

- Users may download and print one copy of any publication from the public portal for the purpose of private study or research.

- You may not further distribute the material or use it for any profit-making activity or commercial gain

If the publication is distributed under the terms of Article $25 \mathrm{fa}$ of the Dutch Copyright Act, indicated by the "Taverne" license above, please 


\title{
Stress response and structural transitions in sheared gyroidal and lamellar amphiphilic mesophases: Lattice-Boltzmann simulations
}

\author{
Nélido González-Segredo* \\ FOM Institute for Atomic and Molecular Physics (AMOLF), P. O. Box 41883, 1009 DB Amsterdam, The Netherlands \\ Jens Harting ${ }^{\dagger}$ \\ Institut für Computerphysik, Pfaffenwaldring 27, D-70569 Stuttgart, Germany \\ Giovanni Giupponi and Peter V. Coveney \\ Centre for Computational Science, Department of Chemistry, University College London, \\ 20 Gordon Street, London WC1H OAJ, United Kingdom \\ (Received 2 September 2005; published 15 March 2006)
}

\begin{abstract}
We report on the stress response of gyroidal and lamellar amphiphilic mesophases to steady shear simulated using a bottom-up lattice-Boltzmann model for amphiphilic fluids and sliding periodic (Lees-Edwards) boundary conditions. We study the gyroid per se (above the sponge-gyroid transition, of high crystallinity) and the molten gyroid (within such a transition, of shorter-range order). We find that both mesophases exhibit shear thinning, more pronounced and at lower strain rates for the molten gyroid. At late times after the onset of shear, the skeleton of the crystalline gyroid becomes a structure of interconnected irregular tubes and toroidal rings, mostly oriented along the velocity ramp imposed by the shear, in contradistinction with free-energy Langevindiffusion studies which yield a much simpler structure of disentangled tubes. We also compare the shear stress and deformation of lamellar mesophases with and without amphiphile when subjected to the same shear flow applied normal to the lamellae. We find that the presence of amphiphile allows (a) the shear stress at late times to be higher than in the case without amphiphile, and (b) the formation of rich patterns on the sheared interface, characterized by alternating regions of high and low curvature.
\end{abstract}

DOI: 10.1103/PhysRevE.73.031503

PACS number(s): 83.50.-v, 83.80.Qr, 61.30.Cz, 61.30.Jf

\section{INTRODUCTION}

The study of the response to shear in amphiphilic mesophases has been the subject of attention for numerical modelers only in recent years. The interest in the subject is sustained not only by the wide range of applications in materials science and chemical engineering, but also by the need to gain a fundamental understanding of the universal laws governing the self-assembly processes and competing mechanisms present.

Hitherto, studies have focused mainly on the structural changes induced by steady and oscillatory shear, near and far from critical points, in polymer systems [1-6]. The morphologies studied include cubic- and wormlike-micellar, lamellar, and hexagonally-packed-tubular mesophases; more complex structures are the so-called bicontinuous mesophases, of which those liquid-crystalline of cubic symmetry have thus far been considered in far less detail.

The amphiphilic gyroid $[14,15]$ is a bicontinuous cubic liquid crystal consisting of multi-or monolayer sheets of selfassembled amphiphile dividing two regions, each containing phases which are mutually immisicible, e.g., aqueous and hydrocarbon species. These sheets or labyrinths form a triply periodic minimal surface (TPMS) whose unit cell is of cubic

\footnotetext{
*Email address: nelido@amolf.nl

†Email address: j.harting@ica1.uni-stuttgart.de

*Email address: p.v.coveney@ucl.ac.uk
}

symmetry, has zero mean curvature, with no two points on it connected by a straight segment, and has no reflection symmetries. Their skeletons, i.e., the locus bounded by the TPMS, for each immiscible phase, form double (interweaving), chirally symmetric threefold coordinated lattices. There are lyotropic $[14,15]$ and thermotropic transitions between the gyroid and the microemulsion mesophase, the latter being a bicontinuous mesophase of short-range order. The morphologies in the crossover regions of these transitions show shorter-range order than the gyroid's and longer-range order than the microemulsion's, for which reasons they are termed "molten gyroids."

Bicontinuous cubic mesophases of monoglycerides and the lipid extract from the archebacterium Sulfolobus solfataricus have been found at physiological conditions in cell organelles and physiological transient processes such as membrane budding, cell permeation, and the digestion of fats [7]. They can also be synthesized for important applications in membrane protein crystallization, controlled drug release, and biosensors $[8,9]$.

The purpose of this paper is to report on the response to shear of gyroid $(\mathrm{G})$, molten-gyroid $(\mathrm{MG})$, and lamellar $\left(\mathrm{L}_{\alpha}\right)$ amphiphilic mesophases simulated using a bottom-up kinetic-theoretic model for fluid flow. The model is based on a lattice-Boltzmann (LB) method, which has proved to be a modeling tool alternative to and more efficient and robust than sophisticated methods based on continuum equations. This LB method adheres to a bottom-up complexity paradigm [13] in the sense that it is simple and fully particulate 
and no hypotheses of desirable macroscopic behavior are imposed on the microdynamics - yet we have shown in the past its ability to simulate correct segregation kinetics for immiscible fluids [20] and nonequilibrium self-assembly into amphiphilic mesophases $[14,15]$. Knowing that such a simple model is capable of simulating these kinetic processes from a purely bottom-up dynamics, in this paper we investigate how hydrodynamic interactions couple with self-assembly and modify the stability and morphology of the mesophases. The model also has the capability to reproduce morphological transitions without having to assume a macroscopic, freeenergy model, used in other LB methods $[3,10]$ to compute the diffusive currents substantiating self-assembly.

In addition, since our method models amphiphilic molecules as point dipoles - the simplest possible particulate model for an amphiphile - the rheological features emergent from it are expected to be universal for a broad range of amphiphilic systems. Finally, most of the numerical studies measuring the stress response of complex fluids to shear reported in the literature deal with phase-segregating fluids on one side [10], and the more complicated polymeric [11] and glassy systems on the other [12]. In this respect, the present paper stands somewhere in between these two.

Our paper is structured as follows. In the next section we briefly introduce the model and describe the boundary conditions for the imposition of shear. In Sec. III we report on simulation data and conclude that shear thinning occurs for both $\mathrm{G}$ and MG mesophases leading to a transition to a mesophase consisting of tubular and ringlike structures as the strain increases. In Sec. IV we reveal how the presence of an amphiphile in lamellar mesophases induces the formation of rich interfacial patterns surviving shear and allows higher values of stress than in lamellar mesophases without amphiphile. Finally we provide our conclusions in Sec. V.

\section{THE MODEL AND THE LEES-EDWARDS BOUNDARY CONDITIONS}

We utilized an existing bottom-up lattice-Boltzmann model for amphiphilic fluids $[14,15]$, extended to simulate shear flow by means of Lees-Edwards boundary conditions [16]. The model is in turn based on an extension made to the Shan-Chen bottom-up LB model for immiscible fluids to model amphiphilic-fluid flow, and employs 25 microscopic velocities, of speeds 0,1 , and $\sqrt{2}$, in three dimensions (D3Q25 lattice) $[17,18]$. The model uses a BGK (BhatnagarGross-Krook, or relaxation-time) approximation to the collision term of the Boltzmann equation for fluid transport, which allows us to simulate, for large enough lattices [19], the Navier-Stokes (NS) momentum-balance equation in the bulk of each immiscible fluid species, namely, "oil" [or "red" (r)] and "water" [or "blue" (b)]. The model allows the simulation of correct phase-segregation kinetics in the absence [20] and presence [15] of a third, amphiphilic [surfactantlike (s)] dipolar species. The model controls the interparticle forces between $\mathrm{r}, \mathrm{b}$, and $\mathrm{s}$ species via coupling parameters $\left(g_{\text {br }}, g_{\text {bs }}, g_{\text {ss }}\right)$, and transients are controlled via relaxation times for densities $\left(\tau^{\mathrm{b}}, \tau^{\mathrm{r}}, \tau^{\mathrm{s}}\right)$ with an additional relaxation time for the orientation of the amphiphile dipoles $\left(\tau^{d}\right)$. In addition, the model simulates the nonequilibrium selfassembly and relaxation dynamics of sponge $\left(\mathrm{L}_{3}\right)$ and gyroid mesophases $[14,15]$. The gyroids that it simulates show rigidity, arising from their crystalline ordering, which decreases as the concentration of amphiphile is reduced; indeed, a lyotropic transition causes the correlation length to decrease toward that of a sponge mesophase through a molten-gyroid state. This idea is central to the work we present here: we shall see that the mesophase's crystalline ordering enhances its stress response; indeed, we find shear thinning to occur at higher strain rates for gyroids than for sponges.

The Lees-Edwards boundary conditions (LEBCs) were originally proposed by Lees and Edwards in the context of molecular dynamics simulations [16]. They showed that these boundary conditions would give rise to a desired linear, wedged velocity profile while avoiding the troublesome spatial inhomogeneities appearing when solid walls are used to induce the shear flow [21]. A particular realization of the LEBCs on a Cartesian simulation box $\left[0, N_{x}\right] \times\left[0, N_{y}\right]$ $\times\left[0, N_{z}\right]$ is established by letting the periodic images, at $N_{x}<x \leqslant 2 N_{x}$ and $-N_{x} \leqslant x \leqslant 0$, move parallel to unit vectors $\pm \hat{\mathbf{z}}$, respectively, both with speed $U$. The LEBCs, in their original, molecular dynamics form, are expressed as a Galilean transformation on the position $(x, y, z)$ and velocity $\left(\xi_{\mathrm{x}}, \xi_{\mathrm{y}}, \xi_{\mathrm{z}}\right)$ coordinates of a molecule, as follows:

$$
\begin{gathered}
x^{\prime} \equiv x \bmod N_{x}, \\
y^{\prime} \equiv y \bmod N_{y}, \\
z^{\prime} \equiv \begin{cases}\left(z+\Delta_{z}\right) \bmod N_{z}, & x>N_{x}, \\
z \bmod N_{z}, & 0 \leqslant x \leqslant N_{x}, \\
\left(z-\Delta_{z}\right) \bmod N_{z}, & x<0, \\
\xi_{x}^{\prime} \equiv \xi_{x}, \\
\xi_{y}^{\prime} \equiv \xi_{y},\end{cases} \\
\xi_{z}^{\prime} \equiv \begin{cases}\xi_{z}+U, & x>N_{x}, \\
\xi_{z}, & 0 \leqslant x \leqslant N_{x}, \\
\xi_{z}-U, & x<0,\end{cases}
\end{gathered}
$$

where $\Delta_{z} \equiv U \Delta t$ is the image's shift at time $\Delta t$ after the onset of shear.

An implementation of the LEBCs on our LB dynamics (LB LEBCs) differs from that used in molecular dynamics (MD LEBCs) in that the shift $\Delta_{z}$ is not in general a multiple of the lattice unit, as Wagner and Pagonabarraga have pointed out [21], and hence an interpolation scheme is needed. This interpolation scheme streams the amphiphile dipoles $\mathbf{d}(\mathbf{x})$ and mass densities $n_{k}^{\alpha}(\mathbf{x})$ located at position $\mathbf{x}$ on the shearing wall, where $\mathbf{c}_{k}$ is the relevant discrete molecular velocity, $k=1, \ldots, 25$, for each (fluid and amphiphilic) species $\alpha$.

In our LB LEBCs, while the spatial displacement follows Eqs. (1), the velocity shift cannot be enforced by replacing the continuum velocity component $\xi_{z}$ in Eqs. (2) with the 
discrete microscopic speeds $\mathbf{c}_{k} \cdot \hat{\mathbf{z}}$, since the velocities $\mathbf{c}_{k}$ are constant vectors. Instead, this acceleration is enforced on the macroscopic fluid velocity around which the local Maxwellian distributes molecules at equilibrium and toward which the BGK scheme relaxes, similarly to how immiscibility forces are implemented $[15,20]$. This procedure guarantees that all accelerations in the fluid are ruled by the same BGK process, controllable via the shape of the distribution function and the relaxation-time parameter, including the acceleration due to the shearing walls.

The MD LEBCs give rise, at steady state (late times), to a shear state which is Galilean invariant, i.e., no particular plane in the system is favored over another. This is a sine qua non for any shearing method, and our method satisfies it too. As regards the unsteady, transient initial states, the MD LEBCs are unphysical since they cannot provide the molecular specificity (e.g., wall roughness) required in an atomistic approach to boundary effects, such as density layering and slip at wall. However, mesoscopic methods-the LB method is one of them-in general only describe low wave numbers and frequencies, which means that, with respect to MD, (a) the atomistic detail of the shearing walls is largely coarse grained and (b) the fluid structure and dynamics are much less sensitive to the atomistic detail of the walls. Since most boundary effects present in MD are absent in the LB method, the fact that the LE boundary conditions eliminate them does not pose a problem. This should be taken with a caveat: our gyroidal mesophases melt when placed in a solid box, which means that the approach to equilibrium is sensitive to momentum transfer with the walls, and therefore the LE boundary conditions do not mimic shearing a mesophase in confinement. (To our knowledge, no bottom-up simulations have ever reported mesophase self-assembly in confinement.) Rather, the LB LEBCs mimic walls located far enough from where observables are probed that microscopic boundary effects are absent.

Our LEBC implementation is embedded within an efficient parallel LB algorithm [22] which allows us to employ large lattices and hence reach the small Knudsen number limit where (a) regions away from interfaces satisfy the incompressible NS equation in the limit of low Mach numbers (Ma) [20], and (b) observables vary by less than $10 \%$ when the lateral lattice dimension is doubled. We have previously found that the lattice size guaranteeing condition (b) is $128^{3}$ for the parameters generating the mesophases investigated here $[14,15]$.

\section{SHEARING GYROIDAL MESOPHASES}

We sheared two gyroidal mesophases differing in the amount of amphiphile present and the value of the interamphiphile interaction coupling parameter. Each of these structures was allowed to self-assemble from homogeneous mixtures of oil, water, and amphiphile using periodic boundary conditions. They have been appropriately characterized by probing direct and Fourier-space late-time snapshots of the density order parameter $\phi \equiv \rho^{\text {oil }}-\rho^{\text {water }}$; more precisely, they correspond to gyroid [cf. Fig. 5(a)] and molten gyroid mesophases, as previously reported by us $[14,15]$.
The common parameters used for both gyroids were oil and water densities flatly distributed in the range $0<n^{(0) \mathrm{b}}$ $=n^{(0) \mathrm{r}}<0.7$, coupling strengths $g_{\mathrm{br}}=0.08, g_{\mathrm{bs}}=-0.006$, relaxation times $\tau^{\mathrm{b}}=\tau^{\mathrm{r}}=\tau^{\mathrm{s}}=\tau^{\mathrm{d}}=1$, and, for the amphiphile's dipoles, $\beta=10$ and $d_{0}=1$.

Their differing parameters were surfactant densities, flatly distributed in the initial homogeneous mixture, in the ranges $0<n^{(0) \mathrm{s}}<0.9$ for the gyroid and $0<n^{(0) \mathrm{s}}<0.6$ for the molten gyroid, with coupling strengths $g_{\mathrm{ss}}=-0.0045$ for the gyroid and $g_{\mathrm{ss}}=-0.003$ for the molten gyroid. These values for the gyroid are 50\% higher than those for the molten gyroid.

While the gyroid relaxes to a highly crystalline structure [23], the molten gyroid shows both shorter-range order and stronger temporal fluctuations than the former [15]. In order to obtain a sufficiently relaxed molten gyroid as an initial condition for the shear, we took the structure as evolved up to time step 32500 ; regarding the gyroid, the time slice chosen was time step 15000 . For practical reasons, instead of letting the molten gyroid self-assemble starting from a homogeneous initial mixture, we upscaled a smaller molten gyroid, previously self-assembled using the same parameters on a $64^{3}$ lattice [15], to a $128^{3}$ lattice. Upscaling consisted in replicating identical copies of the system: the periodic boundary conditions used to generate the $64^{3}$ system (a) guarantee that the density field is smooth across the replica boundaries, yet, for this same reason, (b) produce a molten gyroid with an additional, undesirable long-wavelength fluctuation whose periodicity is half the lattice size. The amplitude of this undesired long-wavelength fluctuation relaxes in time to a vanishingly small value, a fact which provides us with the $128^{3}$ mesophase we seek. We observed, however, that this relaxation takes place in fewer than 1000 time steps [23], i.e., it is a fast transient which, therefore, does not affect the shear response at the late times that we are interested in. In other words, the late-time shear response is insensitive to a small perturbation in the initial condition. This allowed us to take the upscaled, unrelaxed structure as the initial condition for the molten gyroid.

It is worth noting that we did not require an elongated aspect ratio for the lattices along the direction parallel to the translation of the shearing walls since spatial density fluctuations were much smaller than the lattice size. This is not the case when shearing phase-segregating fluids without an amphiphilic, growth-arresting species, as has been previously reported using LB lattices of up to 128:128:512 sizes and aspect ratio [24].

\section{A. Stress response and transients}

Shear thinning is said to occur when the shear viscosity drops as the strain rate increases. For structured fluids such as those we study in this paper, the dynamic shear viscosity $\eta$ is not expected to be a constant of the strain rate $\dot{\gamma} \equiv \frac{1}{2}\left(\partial_{x} u_{z}+\partial_{z} u_{x}\right)$ as is true of Newtonian fluids, for which

$$
P_{x z}= \pm 2 \eta \dot{\gamma}, \quad \eta \neq \eta(\dot{\gamma}) .
$$

Here $P_{x z}$ is one off-diagonal component of the pressure (or stress) tensor, and the sign, by convention, indicates that the pressure is exerted by the fluid element on the surroundings 


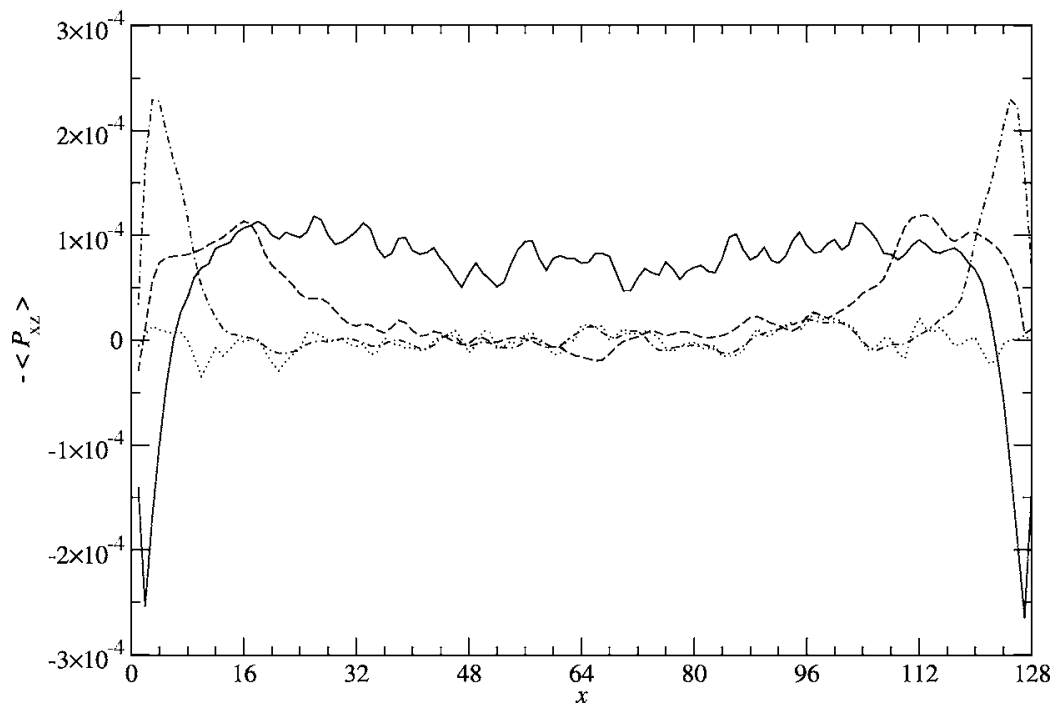

FIG. 1. Shear stress response of a gyroid mesophase along the direction of the velocity gradient. As initial condition, we have taken a gyroid on an $N_{x} N_{y} N_{z}=128^{3}$ cubic lattice at time step $t=15000$ of self-assembly [14,15]. The Lees-Edwards walls move with speed $U=0.10(\mathrm{Ma}=0.17)$. For each $x$ coordinate, the original field has been averaged on the plane $\left[1, N_{y}\right] \times\left[16, N_{z}-16\right]$, where the excluded interval on the $z$ axis accounts for wrapped-round densities. Standard errors of the averages are about $6 \times 10^{-8}$ throughout, and are not shown. Each line represents the response at $\Delta t$ time steps after the start of steady shear: $\Delta t=0$ (dotted line), 100 (dashed-dotted), 800 (dashed), and 9000 (solid), where the last is ca. the time at which the core (i.e., the plane $x=64$ ) fully responds. From the figure we can see that momentum transfer decreases as it reaches the core from the walls. Also, note that the stress inverts its sign at late times adjacent to the boundaries, $\left|x-x_{0}\right| \leqslant 2\left(x_{0}=0,128\right)$. All quantities reported are in lattice units.

$(+)$ or from the latter on the former $(-)$, respectively. We adhere in this paper to the second case. In our simulations, we apply the steady shear described in Sec. II, i.e., the shear is generated by the two image cells of the LB lattice located along the $x$ axis moving in opposite directions. As a consequence, $\partial_{x} u_{z}$ becomes the only nonvanishing component of the velocity gradient, which is also true for the $P_{x z}$ component of the stress tensor (and $P_{z x}$, since the physical requirement that the vorticity $W \equiv \frac{1}{2}\left(\partial_{x} u_{z}-\partial_{z} u_{x}\right)$ remains upper bounded requires the stress tensor to be symmetric).

As we have likewise done previously while computing diagonal components of the pressure tensor [14,15,20], here we measured $P_{x z}$ from its definition as the sum of a kinetic term plus a virial mean-field term accounting for interactions and giving rise to nonideal gas behavior, namely,

$$
\begin{aligned}
\mathbf{P}(\mathbf{x}) \equiv & \sum_{\alpha} \sum_{k} \rho_{k}^{\alpha}(\mathbf{x})\left[\mathbf{c}_{k}-\mathbf{u}(\mathbf{x})\right]\left[\mathbf{c}_{k}-\mathbf{u}(\mathbf{x})\right] \\
& +\frac{1}{4} \sum_{\alpha, \bar{\alpha}} g_{\alpha \bar{\alpha}} \sum_{\mathbf{x}^{\prime}}\left[\psi^{\alpha}(\mathbf{x}) \psi^{\bar{\alpha}}\left(\mathbf{x}^{\prime}\right)+\psi^{\bar{\alpha}}(\mathbf{x}) \psi^{\alpha}\left(\mathbf{x}^{\prime}\right)\right] \\
& \times\left(\mathbf{x}-\mathbf{x}^{\prime}\right)\left(\mathbf{x}-\mathbf{x}^{\prime}\right),
\end{aligned}
$$

where $\psi$ has the form $\psi \equiv 1-\exp [-n(\mathbf{x})]$, which saturates at high density values in order to avoid unbounded interparticle forces while reproducing a meaningful equation of state [15]. Since the interaction matrix $\left\{g_{\alpha \bar{\alpha}}\right\}$ is symmetric with all diagonal elements identically zero, and only nearest neighbor interactions are being considered, the virial term reduces to

$$
\frac{1}{2} \sum_{\alpha \neq \bar{\alpha}} g_{\alpha \bar{\alpha}} \sum_{k} \psi^{\alpha}(\mathbf{x}) \psi^{\bar{\alpha}}\left(\mathbf{x}+\mathbf{c}_{k}\right) \mathbf{c}_{k} \mathbf{c}_{k}
$$

In the incompressible, low-Mach-number limit, our LB model reproduces the NS equation away from interfaces $[17,19]$, which describes a Newtonian fluid with a viscosity being a well-known function of the relaxation time. The presence of an interface, characterized by an interfacial tension and a bending rigidity, however, introduces anisotropies in the fluid's stress tensor which can be accounted for by a tensorial effective viscosity. Since the interface may move, at a speed growing with the strain rate, these anisotropies can become unsteady. Our aim is then to measure how this viscosity evolves with the strain and the strain rate.

In order to probe the function $\eta=\eta(\dot{\gamma})$ for both gyroidal mesophases, we measured $P_{x z}$ for a number of different applied shear rates. The chosen values for $U$ were such that they remained within the incompressibility limit, i.e., small compared to the speed of sound on the D3Q25 lattice, $c_{\mathrm{s}}=3^{-1 / 2} \approx 0.58$. Values chosen were $U=0.05,0.10,0.15$, 0.20 , corresponding to Mach numbers $\mathrm{Ma} \equiv U / c_{\mathrm{s}}=0.086$, $0.17,0.26,0.34$, respectively. All observables we report in this paper are spatial averages, at least on $x=$ const. planes where a simple fluid under the same shear would show translational symmetry for the velocity field, i.e., perpendicular to the velocity gradient. Since, for reasons of computational cost, we do not perform averages over the seed used to generate the pseudorandom initial configuration mimicking a homogeneous ternary mixture, we do not provide error bars around averages.

Figure 1 shows the profile of the stress, for the sheared 


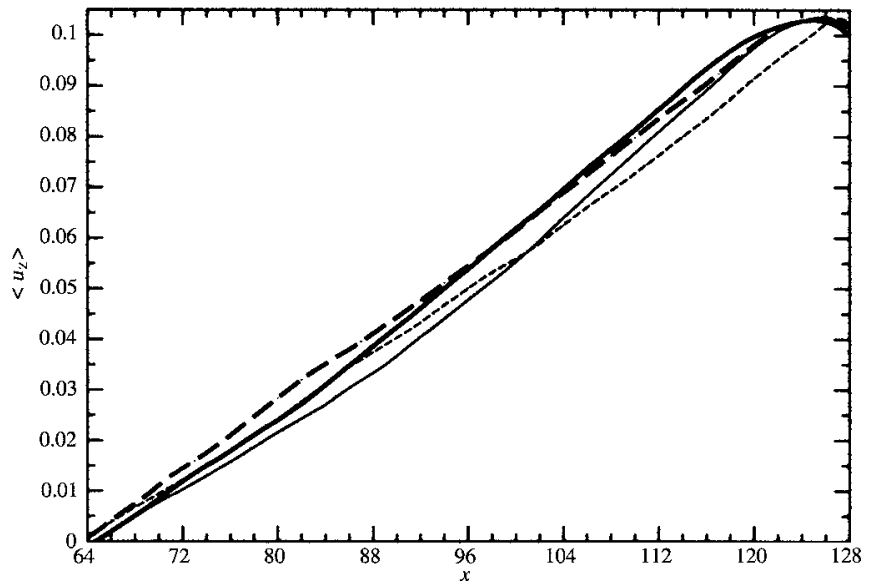

FIG. 2. Spatially averaged velocity component $u_{z}$ for the molten gyroid and the gyroid mesophases sheared with $U=0.10$, at late times and over the $x \geqslant 64$ half of the system. The dashed thin and thick curves correspond to the molten gyroid at time steps $\Delta t$ $=9000$ and 13000 , respectively. The solid thin and thick curves correspond to the gyroid at time steps $\Delta t=9000$ and 13000 , respectively. The average is over the same two-dimensional domain as described in Fig. 1, for each $x$, and its standard error is shown as negligible error bars. Note that the velocity shows a maximum located from two to four sites away from the boundary, unlike a simple fluid which would display it exactly at the boundary. The value of this maximum coincides with the actual velocity at which the BGK relaxation process of our LB model is forcing the fluid to move, which need not coincide with the input parameter $U=0.10$. Note that the inversion in the sign of the stress that we reported in Fig. 1 occurs precisely for $\left|x-x_{0}\right| \leqslant 2, x_{0}=0,128$ and at (late) times close to and after $\Delta t=9000$. The behavior at the other boundary region is similar and symmetric to that displayed here. All quantities reported are in lattice units.

gyroid, along the applied velocity gradient direction. Several curves therein depict the transport of momentum toward the core (i.e., the plane $x=64$ ) as the strain grows as a function of time. Distinctively, the profiles have spatial fluctuations, a consequence of the gyroid's convoluted structure whose interfacial tension locally modifies the viscosity expected for a simple fluid. The $u_{z}$ component of the velocity field, shown in Fig. 2 and averaged in the same way as stated for $\left\langle-P_{x z}\right\rangle$ in the caption of Fig. 1, is, however, not inhomogeneous but follows a transient similar to that expected for a simple fluid: we observe the setting up of a steady, smooth, and wedgeshaped profile, except at the borders. Figure 2 also includes the behavior of the averaged velocity profile for the molten gyroid MG at late times, and is seen to match that of the gyroid $\mathrm{G}$.

Remaining with the $\mathrm{G}$ mesophase, we show in Fig. 3 the temporal evolution of the stress displayed in Fig. 1; the values plotted are averages of the latter on the $8 \leqslant x \leqslant N_{x}-8$ $=120$ interval, which amounts to averaging over the whole lattice except for thin slabs adjacent to the boundaries. In addition to Fig. 1, we include higher and lower shear velocities, namely, $U=0.05,0.15,0.20$. Note that the time evolution of the averaged stress is a succession of peaks and troughs, denoting successive intervals of yield and recoil, which is a canonical feature of viscoelastic behavior. Were the strain rate at which the gyroid deforms coincident with the applied shear rate, these curves would imply shear thinning. In fact, while the increments in applied shear rate between these curves are kept constant, the increments in the (absolute) values of the stress at late times do not remain so but decrease. In Fig. 4 we show the stress averaged over time steps 24000 to 28000 , plotted against the true strain rate, where the latter was measured from the linear velocity profile generated at $\Delta t \geqslant 9000(t \geqslant 24000)$, as displayed in Fig. 2. Figure 4 clearly shows shear thinning: the slope, i.e., the effective viscosity $\eta^{\text {eff }} \equiv \partial P_{x z} / \partial \dot{\gamma}$, decreases with increasing strain rate. Figure 4 also contains the analogous curve for the molten gyroid, which shows shear thinning for the latter at lower strain rates than those at which the gyroid does, and at higher intensity, i.e.,

$$
\left.\frac{\partial \eta^{\text {eff }}}{\partial \dot{\gamma}}\right|_{\text {molten }}<\left.\frac{\partial \eta^{\text {eff }}}{\partial \dot{\gamma}}\right|_{\text {gyroid }}<0 .
$$

No bottom-up kinetic-theoretic model for fluid flow other than ours has hitherto reported an indication of shear thinning.

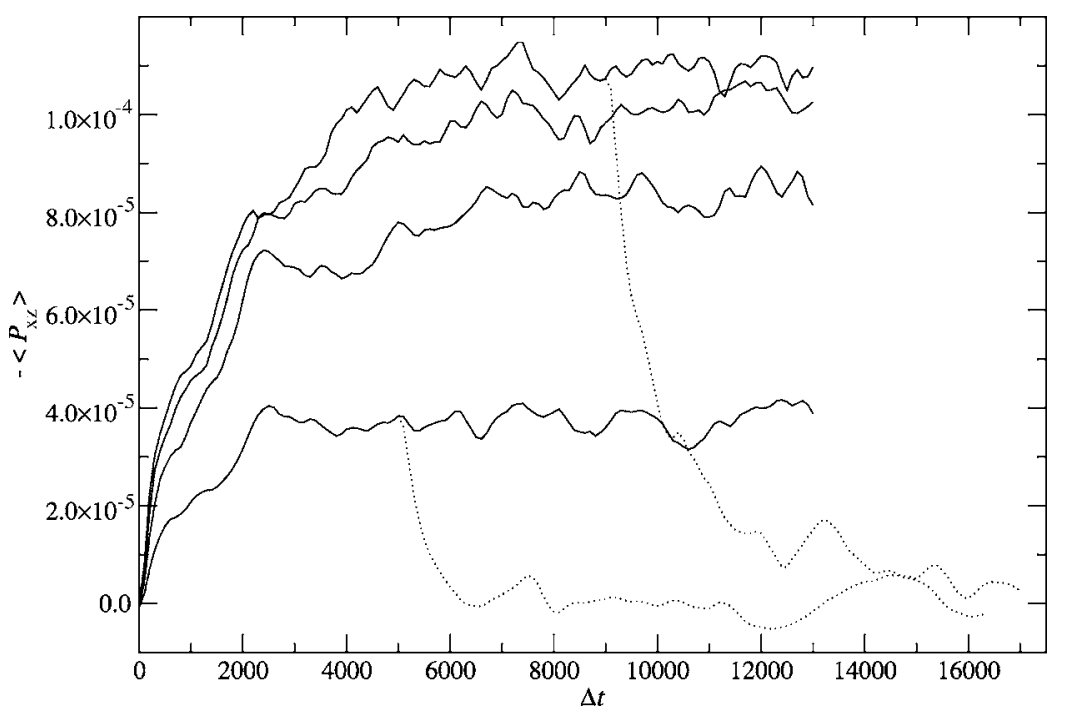

FIG. 3. Temporal evolution of the average shear stress of the gyroid for different values of steady shear. The initial condition is the same as that mentioned in Fig. 1. The curves, as seen, e.g., at $\Delta t=4000$ from bottom to top, correspond to Lees-Edwards walls moving with speeds $U=0.05,0.10,0.15,0.20$ (shear rates $S / 10^{-3}$ $=0.39,0.78,1.17,1.56)$, respectively. The dotted curves are the responses after a sudden termination of shear; they are also referred to as the system's relaxation functions for the relevant shear speeds. The average here is in the three-dimensional domain $\left[8, N_{x}-8\right] \times\left[1, N_{y}\right]$ $\times\left[16, N_{z}-16\right]$, where $N_{x}=N_{y}=N_{z}=128$ and error bars are the standard error of the average. All quantities reported are in lattice units. 


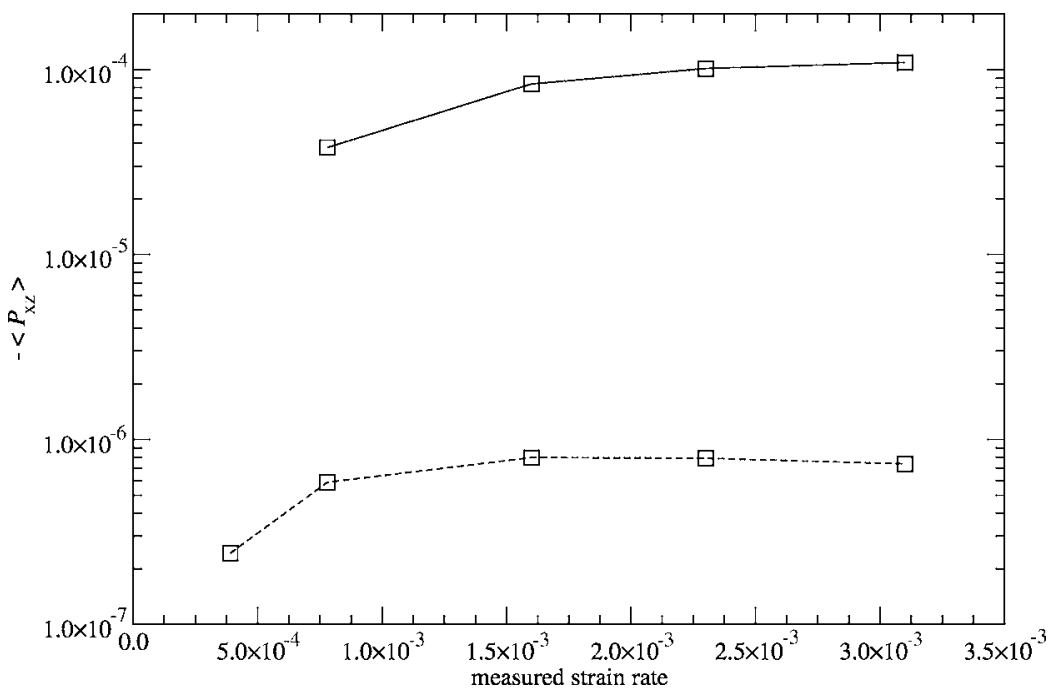

FIG. 4. Both the gyroid (solid line) and the molten gyroid (dashed) mesophases exhibit shear thinning. Shown is the stress averaged over the interval $24000 \leqslant t \leqslant 28000$. From the figure it is clear that the gyroid manifests greater stiffness than the molten gyroid and its (effective) viscosity drops for higher strain rates. All quantities reported are in lattice units.

\section{B. Morphological transitions}

Figure 5 shows the configuration of the gyroid in the 40 $\leqslant y \leqslant 52$ slab of the 128:128:128 lattice, before and at late times after applying a shear of $U=0.20$. The volumerendering graphical representation employed [25] makes regions where $\phi \geqslant 0.37$ opaque to the lighting rays, assumed to shine normal to the plane of the text and inward; since $-0.79 \leqslant \phi \leqslant 0.79$ over the entire system, these regions are the high-density locus of one of the species (say, oil). Before shear, the structure contains highly ordered subvolumes of gyroid symmetry and diagonal length from about 32 to 64 lattice sites [cf. Fig. 5(a)]. This gyroid is hence a collection of subvolumes with a regular tubular structure making up two threefold coordinated, interweaving chiral lattices of which we depict only one. Since the size of the G unit cell is approximately 5-6 lattice units, the depth ( $y$ dimension) of the slabs shown in Fig. 5 is of about two gyroid unit cells. As can be seen in Fig. 5(a), the interfaces between these gyroid subvolumes are defective regions where long-range order and symmetry appear to be drastically reduced $[14,15]$. Two features characterizing them are the spatial variation in coordination number and chirality, seen by the presence of elongated tubules and toroidal rings (cf. Fig. 6).

At $\Delta t=21000$, which is a late time after the onset of shear and we take as steady state, the structure has lost any resemblance to the initial gyroid, except for the persistence of the toroidal rings [see Fig. 5(c)], which are defects in G. Also, the structure at $\Delta t=21000$ is essentially the same as that at time step $\Delta t=5000$ - it is a nonequilibrium steady state for at least the previous 16000 time steps, a time longer than that required for the initial configuration to self-assemble from a homogeneous mixture of oil, water, and amphiphile. The structure at $\Delta t=21000$ consists of an irregular network of mainly the same structural elements characterizing the defective regions before the onset of shear, namely, (a) elongated tubules, with a tendency to align along a direction that is a linear combination of directions $(1,0,0)$ and $(0,0,1)$, and (b) toroidal, ringlike structures. This description is, by visual inspection, similar for every subvolume of the lattice visualized.
We also looked into the structure of the sheared molten gyroid at late times. In contradistinction to the gyroid's state at high strain, showing tubules of shape similar to that depicted in Fig. 6 and at an angle with the $x=$ const planes, the highly strained molten gyroid displays tubes which are more stretched and aligned along the $\hat{\mathbf{z}}$ direction. The toroidal rings, also present for the molten gyroid before shear, represent a much smaller volume fraction for the sheared molten gyroid than for the sheared gyroid.

Figure 7 shows the summed structure function $\Sigma_{k_{y}} S(\mathbf{k}, t)$, or "scattering pattern", of the sheared gyroid mesophase, showing stages of its plastic deformation. Here, $S(\mathbf{k}, t)$ is the structure function, computed according to $[15,20]$

$$
S(\mathbf{k}, t) \equiv \frac{\boldsymbol{s}}{V}\left|\phi_{\mathbf{k}}^{\prime}(t)\right|^{2}
$$

Here, $\mathbf{k}$ is the discrete wave vector, $V$ is the lattice volume, $\mathbf{s}$ is the unit cell volume for the D3Q25 lattice, and $\phi_{\mathbf{k}}^{\prime}(t)$ is the Fourier transform of the fluctuations of $\phi . S(\mathbf{k}, t)$ is the Fourier transform of the autocorrelation function for the order parameter,

$$
C_{\phi \phi}(\mathbf{r}, t) \equiv\langle\phi(\mathbf{x}, t) \phi(\mathbf{x}+\mathbf{r}, t)\rangle
$$

where $\mathbf{r}$ is a vector lag and the angular brackets indicate an average over the spatial coordinate x. Figures 7(a), 7(b), and $7(\mathrm{~d})$ are the $x z$ "scattering patterns" of the structures in Fig. 5 , produced by summing up the structure function along the $x$ direction. At $\Delta t=1000$ (not shown), the maximum intensity is reduced to $29 \%$ of its value at $\Delta t=0$, while there appear horizontal "smeared out filaments" of very weak intensity, intrinsically related to the shearing process, as we shall conclude from Fig. 8. At $\Delta t=5000$ a clear cardioid shape has developed; the fact that it persists for the rest of the simulation confirms our observation that the system reaches a steady state at time step $\Delta t=5000$. In addition, there is no trace of gyroidal patterns along the $x$ direction.

In order to investigate the origin of the cardioid shape, we computed the scattering pattern for a "synthetic gyroid" 

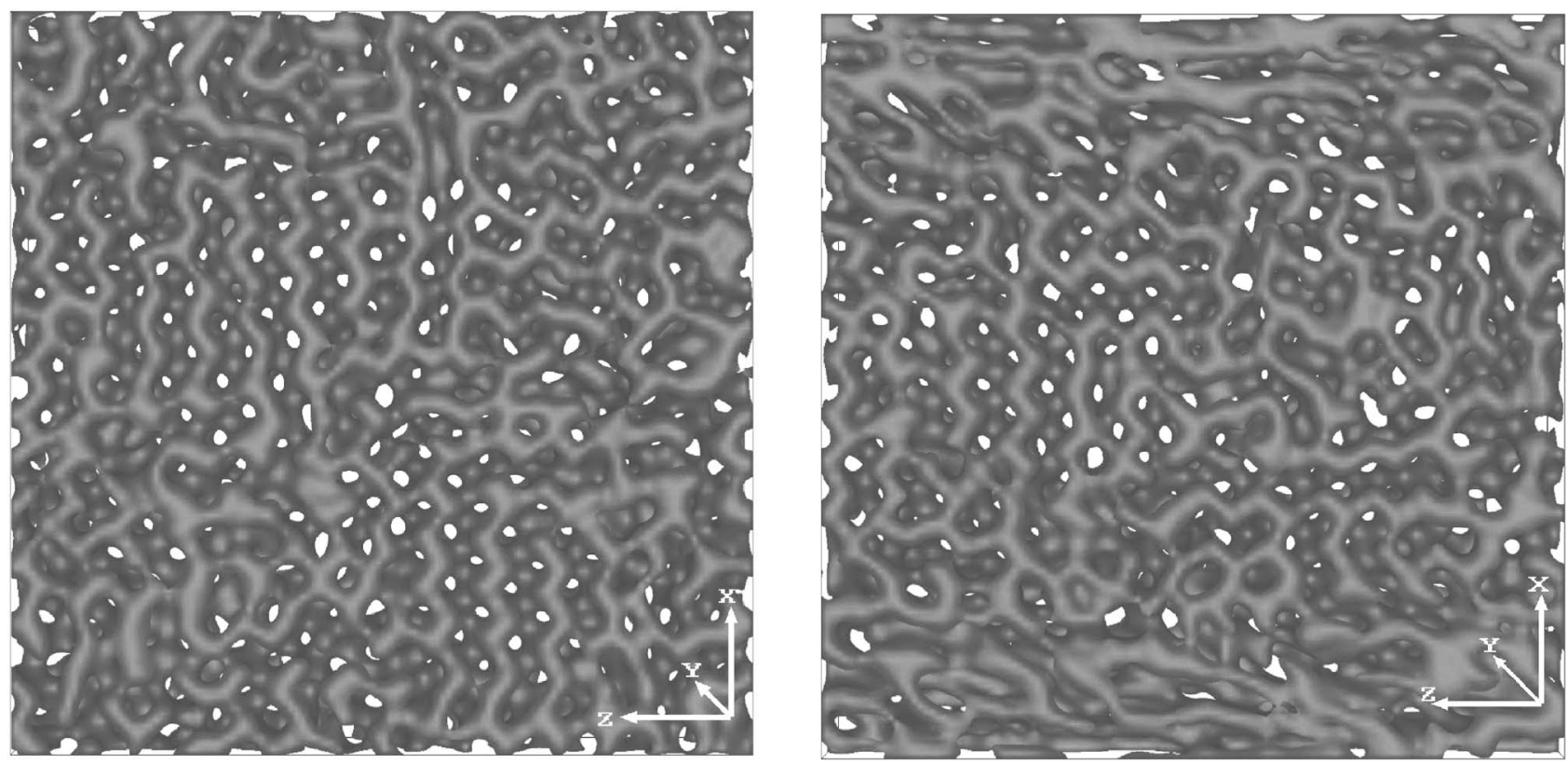

(a) $\Delta t=0$

(b) $\Delta t=1000$

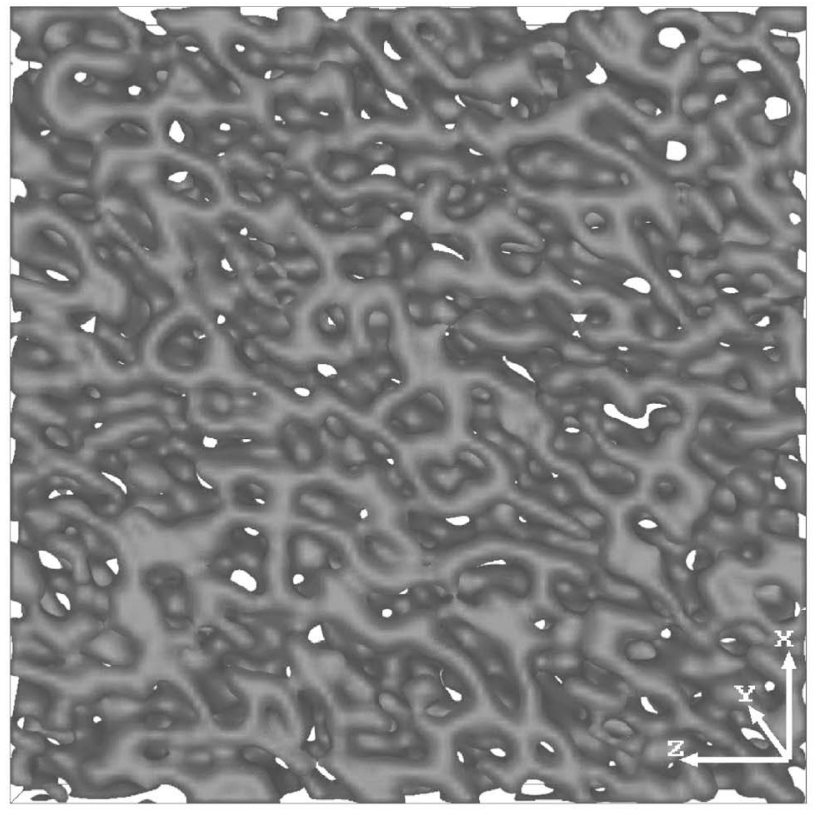

(c) $\Delta t=21000$

FIG. 5. High-density locus of one species (say, oil) in the gyroid mesophase, before shearing (a), and at an early (b) and late time slice (c), after the onset of shear. The shear speed is $U=0.20$. The complementary immiscible fluid (water) fills the voids with a similar, interweaving structure. The system is on a $128 \times 128 \times 128$ lattice, and all figures show the subvolume $40 \leqslant y \leqslant 52$ and the reference system in use (the $y$ axis is perpendicular to the plane of the page). The initial configuration (a) is a gyroid at 15000 time steps of self-assembly under periodic boundary conditions. These images are volume renderings of the density order parameter $\phi \equiv \rho^{\text {oil }}-\rho^{\text {water; }}$ the regions visible to the reader are those for which $\phi \geqslant 0.36$ while over the entire fluid $-0.79 \leqslant \phi \leqslant 0.79$; the regions for which $\phi \leqslant-0.36$ (water, not shown) display a similar structure which is complementary (interweaving) to the one shown here. All quantities reported are in lattice units.

$$
\begin{aligned}
G(\mathbf{x}) \equiv & \sin q x \cos q y+\sin q y \cos [q z-\delta(\mathbf{x})] \\
& +\sin [q z-\delta(\mathbf{x})] \cos q x,
\end{aligned}
$$

where $\delta(\mathbf{x})=\left(x-N_{x} / 2\right) \delta_{\max }$ is a spatially varying dephase used to obtain a linear strain on the morphology (its maximum value $\delta_{\max }$ is reached at the lattice boundaries), and $q=$ const is a wave number controlling the size of the surface's unit cell. It is known that $G(\mathbf{x})=0$ for $\delta_{\max } \equiv 0$ is a 


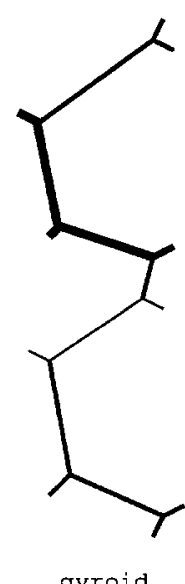

gyroid

FIG. 6. Schematic representation of the skeleton (locus of highest density) of the gyroid mesophase we employ, and two of its structural features before and at late times after the onset of steady shear. The thickness provides a sense of perspective, and represents how close each segment is to the reader; note that the figures on the right are planar. The skeleton denoted by "gyroid" depicts a portion of one of the two chiral lattices making up the long-range order regions of the gyroid before shear [cf. Fig. 5(a)]-the coordination number is three at each node. In the regions of the gyroid containing defects, as well as in most of the sheared mesophase at late times, the coordination number can be reduced to 2 , describing a "tubule." We also show the skeleton of the "ring" structure ubiquitous in the sheared gyroid at late times, also present in smaller proportion as a defect in the mesophase before the onset of shear. At lower values of density, this ring appears to be toroidal.

good approximation to the Schoen "G" triply periodic minimal surface of $I a \overline{3} d$ cubic symmetry, referred to as "the ideal gyroid" hereafter [26]. Figure 8 shows the scattering patterns for the unstrained morphology and for dephases $\delta_{\max }=8,16$.

Comparing structure function maps in Figs. 7 and 8, at the same value of the strain rate, proves useful. For the synthetic gyroid, the strain is controlled by the number of unit cells that the dephase causes the structure to shift at the lattice boundary, following a linear profile as we approach the other boundary going through zero strain at the lattice core. For our simulated amphiphilic gyroid, however, the strain does not follow a linear profile at early times; instead, the strain at time $t$ would need to be computed from the integral $\left(1 / N_{x}\right) \int_{0}^{t} \int_{0}^{N_{x}} \mathrm{~d} t^{\prime} \mathrm{d} x \partial_{x} u_{z}\left(\mathbf{x}, t^{\prime}\right)$, where $t^{\prime}$ is the time parameter. For the purposes of this paper, however, such an analysis would be superfluous; in fact, Fig. 8 already provides us with enough information to understand the origin of the cardioid shape. For all panels (a), (b), and (c) therein, the positions of the peaks at $k_{z}=0\left[k_{x} /(2 \pi / N) \approx-14,15\right.$, where $\left.N=128\right]$ are invariant under the strain (dephase); not so with the peaks at $k_{z} \neq 0$, which shift leftward. (The shift would be rightward were $\partial_{x} u_{z}<0$ or $\delta_{\max }$.) The shape of the maps in Figs. 7(c) and $7(\mathrm{~d})$ is that of a transformed scattering pattern shifted leftward. This transformation occurs early, between $\Delta t=0$ and 3000 , and is characterized by two (strong, $S \geqslant 700$ ) peaks similar to those of the gyroid at $k_{x}=0$, and two (weaker, $200 \leqslant S<700$ ) peaks at $k_{z}=0$.

\section{A SIMPLER CASE: SHEARING THE LAMELLAR MESOPHASE}

In the last section we reported on the gyroid displaying higher shear stress than the molten gyroid. Since the structural transition between these two mesophases can be driven by both the amphiphile density and the interamphiphile coupling parameter, as we have reported in the past [15], our aim in this section is to elucidate the role of the amphiphile density alone on the stress response to shear; we choose the lamellar mesophase as the subject of study, since this is the mesophase with the simplest possible internal interface.

The initial configuration employed was a cubic $128^{3}$ lattice with 16 lamellae, stacked perpendicularly to unit vector $\hat{\mathbf{z}}$. The lamellae were of alternating oil-water compositions, separated by a thin monolayer of amphiphile; the thicknesses of the immiscible and amphiphilic lamellae were 7 and 1 lattice sites, respectively. We populated each lattice site with a value of density kept constant over the region corresponding to a given species; each microscopic velocity is assigned the same fraction of this value. We gave amphiphilic regions the densities $n^{(0) \mathrm{s}}=0,0.80,0.95$, and oil and water regions the densities $n^{(0) \mathrm{r}}=n^{(0) \mathrm{b}}=0.7$. Shear was applied perpendicular to the lamellae with the same LEBCs employed in the last section, with speed $U=0.10$.

Before the onset of shear, the case without amphiphile for the lamellar initial condition just described is, a priori, a metastable state in our LB model. In fact, the structure has a stationary morphology since short-range oil-water forces and the absence of fluctuations maintain immiscibility, i.e., a value for the interface steepness $|\nabla \phi|$; however, a large enough perturbation in $\phi$ may allow a fluctuation in surface tension which drives the entire interface to a radically different shape. Another factor disrupting this lamellar morphology is shear, which may work against the interfacial tension by reducing $|\nabla \phi|$; this can lead to miscibility $(\phi \equiv 0)$ for high enough strain rates. Despite these arguments, we observed stability for the sheared lamellar mesophase without amphiphile, as we report next.

Figure 9 shows the stress as measured in the same fashion performed on the data plotted in Fig. 3, for several amphiphile densities. The behavior observed is diverse. For zero amphiphile concentration (solid curve), the stress reaches a peak at early times before it proceeds to a second, lower maximum at late times, going through a trough at intermediate times due to the fact that $|\nabla \phi|$ experiences a transient decrease.

The high-density regions of one of the immiscible species (say, oil) is shown in Fig. 10(a) at late times, $\Delta t=8000$; these are representative of the shape of the oil-water interface. Away from the boundaries $(x=0,128)$, there is a large interfacial area with zero curvature, where we define the curvature as $H \equiv \partial_{z z}^{2} x_{\phi}(z), x_{\phi}(z)$ being the curve resulting from projecting the $\phi=0.18$ surface onto the $x z$ plane. Curiously, we observe three changes of curvature as we follow the curve $x_{\phi}(z)$ for $y=$ const, namely, $H<0, H>0, H<0, H>0$; instead, we would have expected the steady, late-time configuration for the sheared lamellar mesophase to rather minimize the interfacial area, leaving only one inflection point. 


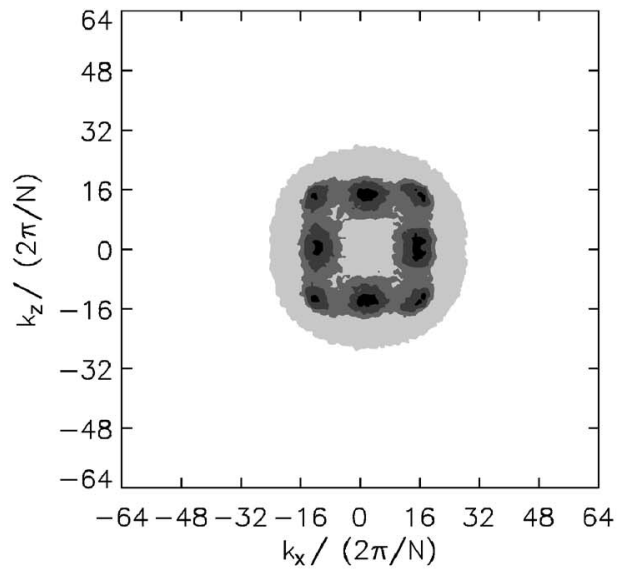

(a) $\sum_{k_{\mathrm{y}}} S(\mathbf{k}, t), \quad \Delta t=0$

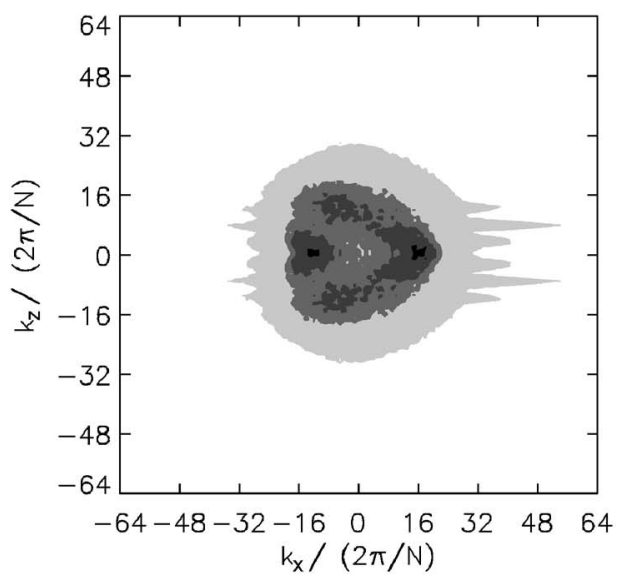

(c) $\sum_{k_{\mathrm{y}}} S(\mathbf{k}, t), \quad \Delta t=21000$

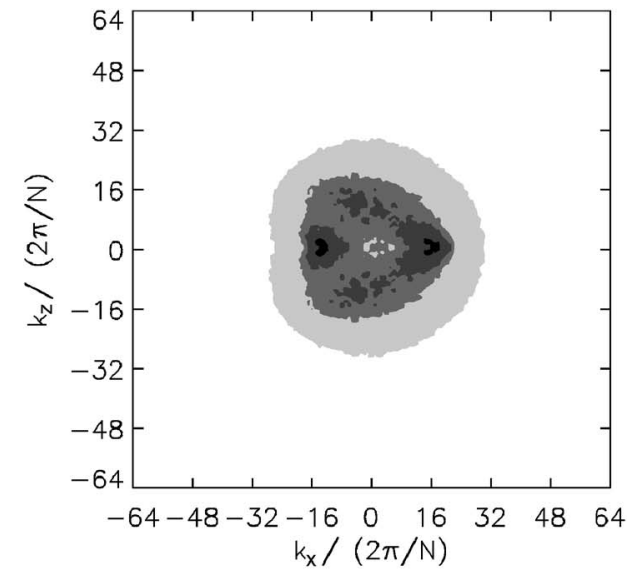

(b) $\sum_{k_{y}} S(\mathbf{k}, t), \quad \Delta t=5000$

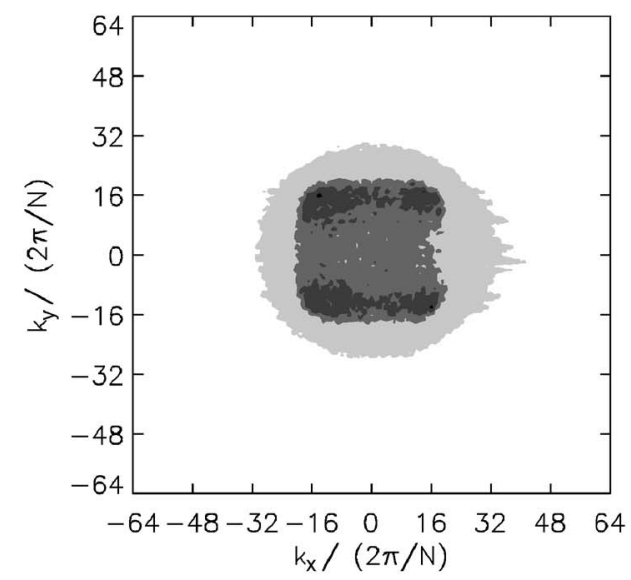

(d) $\sum_{k_{z}} S(\mathbf{k}, t), \quad \Delta t=21000$
FIG. 7. Projected structure function ("scattering pattern", $S$ ) as a function of the time step for the sheared gyroid, as calculated using Eq. (7). Shear velocity is $U=0.20$. (a), (b), and (c) are scattering patterns before shear and at intermediate and late times after the onset of shear, respectively, while, for completeness, (d) details the side view of the structure function corresponding to (c). The initial condition for shearing was a gyroid on a $128^{3}$ lattice at 15000 time steps of selfassembling. Time steps after the start of shear for these snapshots are indicated below each. Darkness in the grayscale grows with the scattering intensity-filled isocurves correspond to values $S=1,80,200,700$. The spikes are shear-dependent features; see Fig. 8 and text for discussion. All quantities reported are in lattice units, and $N \equiv N_{x}=N_{z}$.
For fluid regimes under conditions of local thermodynamic equilibrium, we can think of $H^{2}$ as an interfacial free-energy density associated with the curvature [27]; in this case, we would have expected the steady, late-time configuration to also minimize the interfacial free energy.

The stress curve corresponding to $n^{(0) \mathrm{s}}=0.80$ (cf. Fig. 9) shows the absence of large troughs, as occurs for the $n^{(0) \mathrm{s}}$ $=0$ case, despite the fact that interfacial tension is drastically reduced by the presence of the amphiphile. In addition, the stress grows at late times to higher values than those achieved by the $n^{(0) s}=0$ case. The late-time order-parameter configuration is displayed in Fig. 10(b), showing a rich interfacial pattern. Using the same arguments as those of the last paragraph, this structure could be characterized by a higher curvature energy $\int d^{2} x H^{2}$, where $d^{2} x$ is a measure on the oil-water interface, and $H$ is now defined as the inverse radius of curvature, parametrized on the arclength $s$. Figure 10(b) shows similar regions of high curvature at an equal distance from the shearing walls, where $u_{\mathrm{z}}=$ const, which we shall call nodal planes. Also note that the interface, as approximately depicted by the boundary of the $\phi \geqslant 0.22$ volume, joins the lattice boundary at an angle close to $90^{\circ}$.

The stress curve for the $n^{(0) \mathrm{s}}=0.95$ case shows a dramatically different situation for the first 5000 time steps: the presence of a trough, deeper than that present for the $n^{(0) \mathrm{s}}=0$ density. After that, there appears a shoot-off whereby the stress rapidly grows and equals the late-time value achieved in the $n^{(0) \mathrm{s}}=0.80$ case, while the order parameter displays a configuration analogous to the $n^{(0) s}=0.80$ case [cf. Fig. $10(\mathrm{c})]$. By looking at the amphiphile density field $\rho^{\mathrm{s}}(\mathbf{x})$ for the case $n^{(0) s}=0.95$, we observed that the high-curvature regions arise close to the boundaries first $(\Delta t<1000)$, and then rapidly move away from them as the strain progresses.

\section{CONCLUSIONS}

In this paper we have reported on the shear stress response of two gyroidal cubic amphiphilic mesophases previously self-assembled using the same bottom-up LB model we employ here, namely, the gyroid per se, $\mathrm{G}$, which shows high crystallinity at late self-assembly times, and the molten gyroid MG, endowed with shorter-range order and located within the sponge-gyroid lyotropic structural transition [15]. Shear was imposed via sliding periodic (Lees-Edwards) boundary conditions, and we investigated the response to several values of the strain rate. In addition, in order to investigate the dependence of the shear stress on the amphiphile density, we also sheared a lamellar mesophase, of much simpler morphology than the gyroidal mesophases. 


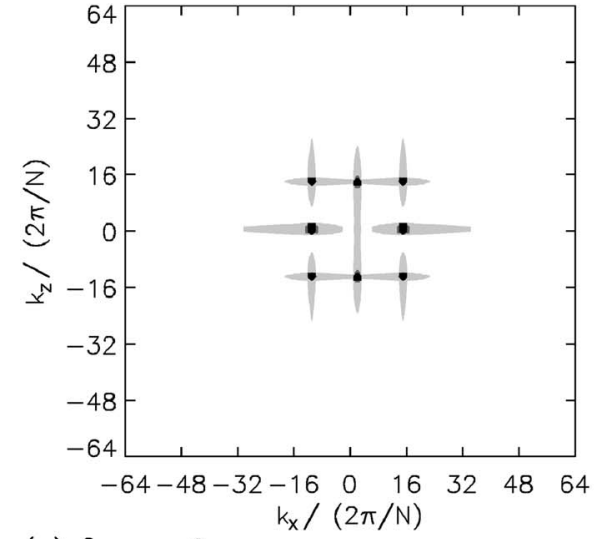

(a) $\delta_{\max }=0$

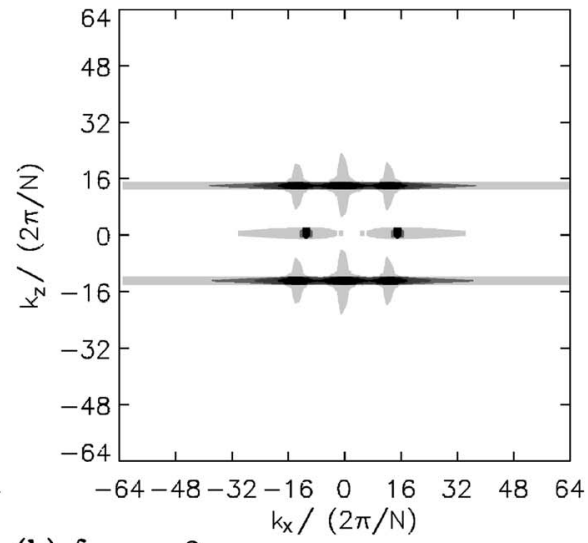

(b) $\delta_{\max }=8$

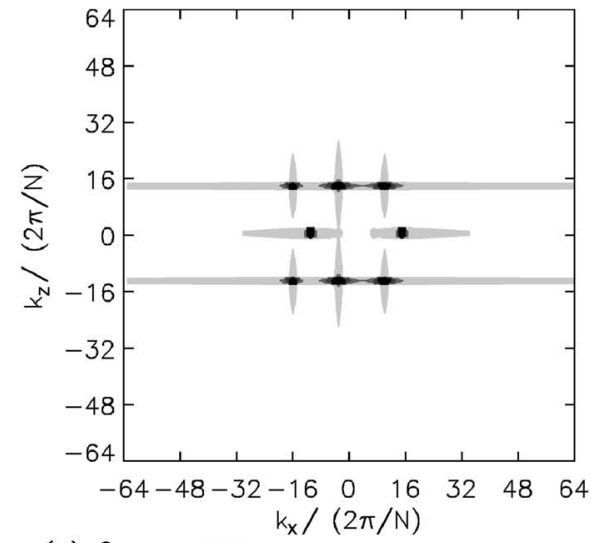

(c) $\delta_{\max }=16$

FIG. 8. Structure function, $S$, of the synthetic gyroid, as calculated using Eq. (7) on the field $G(\mathbf{x})$, the expression of the latter being Eq. (9). Parameter $\delta_{\max }$ is the maximum value of the dephase $\delta(\mathbf{x})=\left(x-N_{x} / 2\right) \delta_{\max }$, which serves to mimic a uniform strain across the structure. The case $\delta_{\max }=0$ gives an approximation to the Schoen G (or ideal gyroid) structure. Darkness in the grayscale grows with the scattering intensity, and the filled isocurves shown correspond to $S=1,80,200,700$. For $k_{z} \neq 0$, the strain shifts the pattern leftward and smears the peaks, while leaving the $k_{z}=0$ peaks intact. The smearing not being in direct relation to the strain-(b) shows more smearing than (c) — suggests a similar behavior for the spikes shown in Fig. 7(c). The cardioid shape reported in Fig. 7 originates from the fact that the structure undergoes a structural transition (weaking and/or relocation of some of its $k_{z} \neq 0, k_{x} \neq 0$ peaks) while being sheared with a velocity profile of positive slope (cf. Fig. 2), which orients the atria leftward. All quantities reported are in lattice units, and $N \equiv N_{x}=N_{z}$.

We found that the gyroidal mesophases exhibit shear thinning, more pronounced and at lower strain rates for the MG mesophase than for the $\mathrm{G}$ mesophase. In other words, momentum introduced into the system due to shear is transported more easily for the mesophase containing more amphiphile, with longer-range ordering, i.e., the effective viscosity is higher for the $\mathrm{G}$ mesophase.

We also found a shear-induced transition from an initial gyroidal morphology ( $\mathrm{G}$ and $\mathrm{MG}$ ) to a mesophase characterized by coexisting elongated tubules and toroidal, ringlike structures. The features of this mesophase are in contrast to those of the mesophase reported by Zvelindovsky et al. using free-energy Langevin-diffusion methods by shearing a bicontinuous structure reminiscent of a molten gyroid [3]. The structure they found is of a shorter-range ordering than that of the MG mesophase described here, and the high-strain structure consists of coexisting lamellae and hexagonally packed tubes elongated along the direction of the imposed shear velocity. Our sheared mesophases also show enlongated tubes along this direction, but the structure is far more complicated than that found by Zvelindovsky et al. in that it exhibits remnant toroidal rings and "hard shoulders" reminiscent of gyroidal skeletons; hexagonal packing and coexisting lamellae are, on the other hand, absent.

The shear performs a plastic deformation which effectively breaks the links of the gyroidal skeleton; this happens as these links interpose an (oil-water) interface whose normal $\mathbf{n}$ is parallel or antiparallel to the flow streamlines $\mathbf{u}$. In other

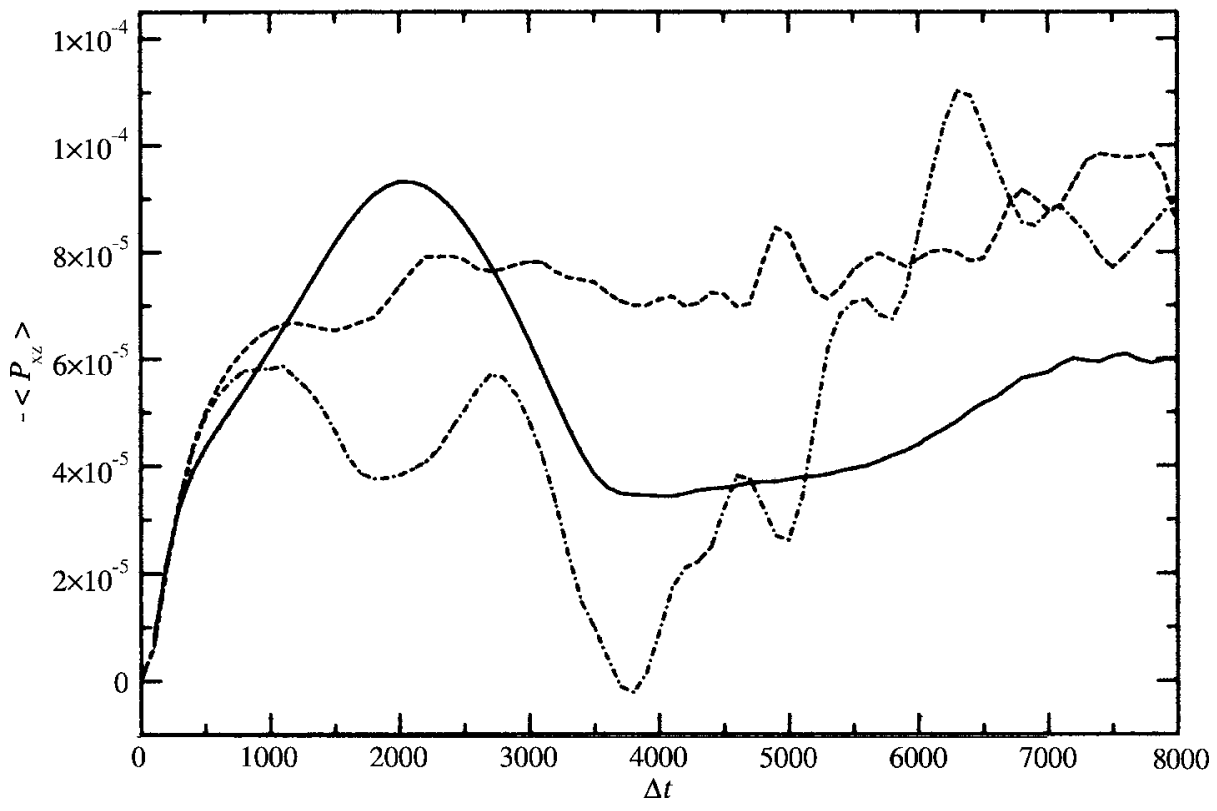

FIG. 9. Temporal evolution of the average shear stress response of a lamellar mesophase at a shear speed of $U=0.10$, for different initial amphiphile densities $n^{(0) \text { s }}$. The solid, dashed, and dashed-dotted curves correspond, respectively, to $n^{(0) \mathrm{s}}=0,0.80,0.95$. The average is computed over the threedimensional domain $\left[8, N_{x}-8\right]$ $\times\left[1, N_{y}\right] \times\left[16, N_{z}-16\right], \quad$ where $N_{x}=N_{y}=N_{z}=128$ and error bars are not included since they are negligible. All quantities reported are in lattice units. 


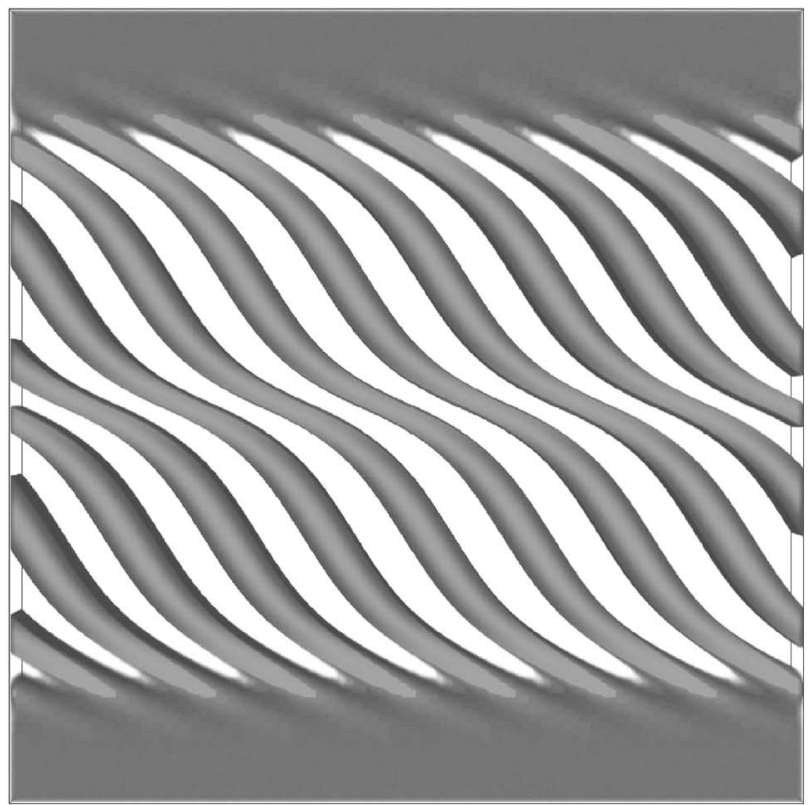

(a) $n^{(0) s}=0$

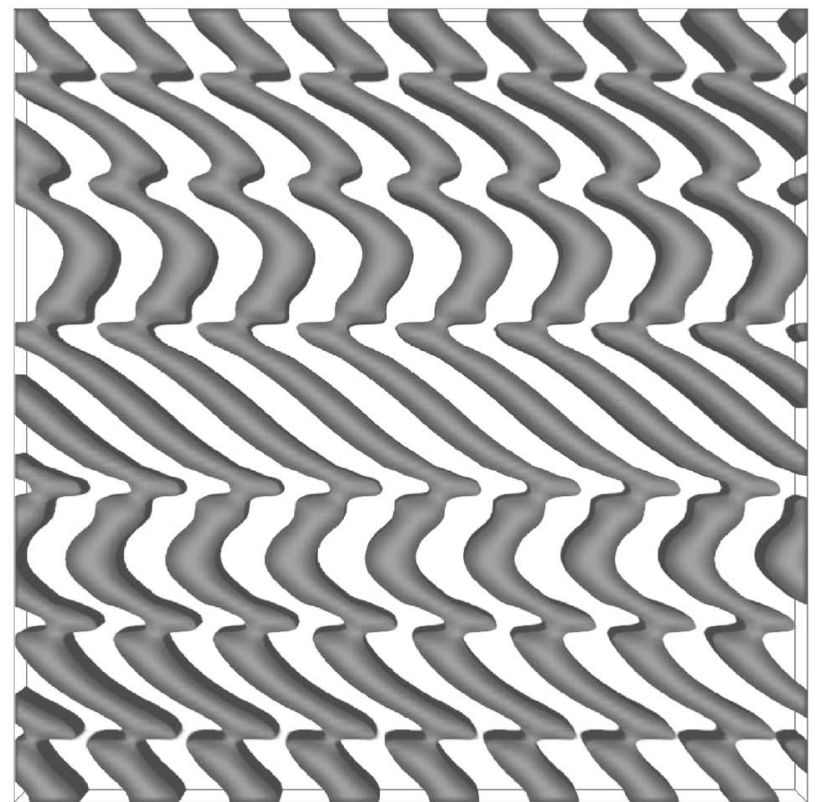

(b) $n^{(0) s}=0.80$

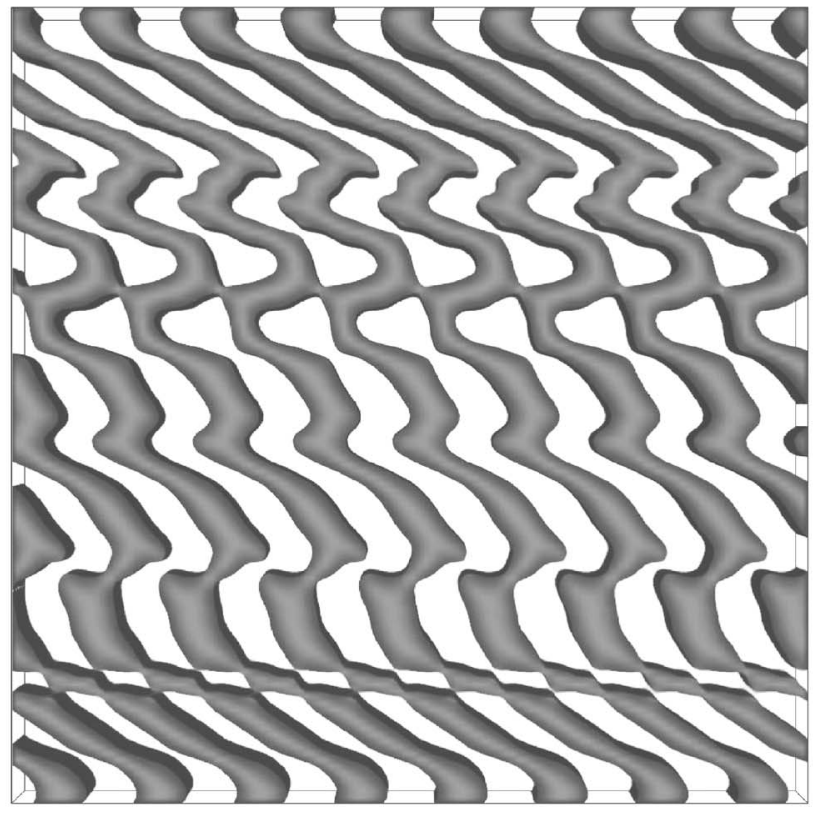

(c) $n^{(0) \mathrm{s}}=0.95$

FIG. 10. Slabs $0 \leqslant y \leqslant 8$ of the order parameter $\phi$ for sheared lamellar mesophases corresponding to increasing amphiphile density $n^{(0) \mathrm{s}}$ as indicated below each relevant panel, at time step $\Delta t=8000$ after the onset of shear and for shear velocity $U=0.10$. The coordinate system is the same as that in Fig. 5. In (a), the regions opaque to incident (volume rendering) light are those for which $\phi \geqslant 0.18$, where $|\phi|$ $\leqslant 0.36$ across the system. In (b), the opaque regions are those for which $\phi \geqslant 0.22$, where $|\phi| \leqslant 0.45$ across the system. In (c), the opaque regions are those for which $\phi \geqslant 0.24$, where $|\phi| \leqslant 0.48$ across the system. It is worth noting that the surfactantless case (a) exhibits a curved interface. The amphiphilic cases (b) and (c) display the formation of irregularities in the interface and nodal planes, as a result of the interamphiphile interaction. All configurations have translational symmetry along the $y$ axis. All quantities reported are in lattice units.

words, shear effectively applies a "mixing" force which is in competition with the interparticle forces keeping the mesophase in place, namely, those controlled by coupling parameters $g_{\text {br }}, g_{\text {bs }}$, and $g_{\mathrm{ss}}$. Our hypothesis is that adsorbed dipoles on interfacial regions at an angle $\theta \equiv \angle(\mathbf{u}, \mathbf{n})$ other than $\theta=0, \pi$ require more work from the shear forces to be drawn away from the interface than those regions on which the streamline impinges normally, since the mixing force goes as $\cos \theta$. In particular, since the mixing force vanishes for $\theta=\pi / 2$, considerably longer interfaces can survive the flow-shear induces a preferential direction along which the long-range order present before the onset of shear is not reduced. This explains not only the formation of the elongated tubules but also their reconnection (increase in coordination 
number). In fact, the toroidal, ringlike structures are not only vestigial gyroid defects which have survived the gradient $\nabla \mathbf{u}$, but are also created anew as a result of reconnections. It is relevant to point out that Padding and Boek, using a coarse-grained molecular dynamics model for wormlike micelles, reported on the formation of rings when applying steady shear to a wormlike micellar mesophase [6] - this is an "amphiphile-in-water" binary mesophase, in contrast to the "oil-amphiphile-water" ternary mesophases we study in this paper.

By applying shear to a lamellar mesophase we found that the presence of amphiphile on the oil-water interface of the mesophase causes the interface to fold into a wealth of structures with a (discrete) translational symmetry on planes equidistant from the shearing walls and along the direction of the shear velocity. In other words, the interamphiphile force couples the adsorbed amphiphilic dipoles so that the interface locally increases its curvature energy density. It is worth investigating whether this local increase is due to the amphiphile being incapable of sustaining interfacial regions of low curvature under shear, i.e., whether the "breaking" mechanism induced by shear is counteracted by regions of high curvature energy density. Regarding the shear stress, our amphiphile-containing lamellae responded with higher stress at late times than those without amphiphile. This contrasts with the results found for the gyroidal mesophases, and lets us conclude that it is the gyroid's cubic morphology that allows this structure to be stiffer. Understanding the behavior of the lamellar mesophase under shear requires the study of amphiphile self-assembly under shear, including in- and outof-plane amphiphilic and associated Marangoni currents, and their coupling to the imposed flow.

\section{ACKNOWLEDGMENTS}

We thank Dr. Rafael Delgado-Buscalioni, Professor Antonio Coniglio, and Professor Francesco Sciortino for enlightening discussions. We acknowledge Iain Murray, Elena Breitmoser, and Jonathan Chin for their involvement in algorithm implementation and optimization within the RealityGrid and TeraGyroid projects. This work was supported by the U.K. EPSRC under RealityGrid Grant No. GR/R67699 which also provided access to a 512-processor SGI Origin3800 platform at Computer Services for Academic Research (CSAR), Manchester Computing, U.K. We also thank the Higher Education Funding Council for England (HEFCE) for our on-site 16-node SGI Onyx2 graphical supercomputer.
[1] A. V. M. Zvelindovsky, B. A. C. van Vlimmeren, G. J. A. Sevink, N. M. Maurits, and J. G. E. M. Fraaije, J. Chem. Phys. 109, 8751 (1998).

[2] A. V. Zvelindovsky, G. J. A. Sevink, B. A. C. van Vlimmeren, N. M. Maurits, and J. G. E. M. Fraaije, Phys. Rev. E 57, R4879 (1998).

[3] A. V. Zvelindovsky, G. J. A. Sevink, and J. G. E. M. Fraaije, Phys. Rev. E 62, R3063 (2000).

[4] A. N. Morozov, A. V. Zvelindovsky, and J. G. E. M. Fraaije, Phys. Rev. E 64, 051803 (2001).

[5] A. N. Morozov and J. G. E. M. Fraaije, Phys. Rev. E 65, 031803 (2002).

[6] J. T. Padding and E. S. Boek, Phys. Rev. E 70, 031502 (2004).

[7] P. Mariani, V. Luzzati, and H. Delacroix, J. Mol. Biol. 204, 165 (1988)

[8] S.-J. Marrink and D. P. Tieleman, J. Am. Chem. Soc. 123, 12383 (2001).

[9] V. Luzzati, R. Vargas, P. Mariani, A. Gulik, and H. Delacroix, J. Mol. Biol. 229, 540 (1993).

[10] A. Xu, G. Gonnella, and A. Lamura, Phys. Rev. E 67, 056105 (2003).

[11] R. D. Groot and W. G. M. Agterof, Macromolecules 28, 6284 (1995).

[12] P. Sollich, Phys. Rev. E 58, 738 (1998).

[13] P. V. Coveney, Philos. Trans. R. Soc. London, Ser. A 361, 1057 (2003).

[14] N. González-Segredo and P. V. Coveney, Europhys. Lett. 65, 795 (2004).

[15] N. González-Segredo and P. V. Coveney, Phys. Rev. E 69, 061501 (2004).
[16] A. W. Lees and S. F. Edwards, J. Phys. C 5, 1921 (1972).

[17] H. Chen, B. M. Boghosian, P. V. Coveney, and M. Nekovee, Proc. R. Soc. London, Ser. A 456, 2043 (2000).

[18] An example of the use of the D3Q25 lattice in lattice-gas methods is given by P. J. Love, P. V. Coveney, and B. M. Boghosian, Phys. Rev. E 64, 021503 (2001).

[19] S. Succi, The Lattice-Boltzmann Equation-For Fluid Dynamics and Beyond (Oxford University Press, Oxford, 2001.)

[20] N. González-Segredo, M. Nekovee, and P. V. Coveney, Phys. Rev. E 67, 046304 (2003).

[21] A. J. Wagner and I. Pagonabarraga, J. Stat. Phys. 107, 521 (2002).

[22] P. J. Love, M. Nekovee, P. V. Coveney, J. Chin, N. GonzálezSegredo, and J. M. R. Martin, Comput. Phys. Commun. 153, 340 (2003).

[23] J. Harting, M. J. Harvey, J. Chin, and P. V. Coveney, Comput. Phys. Commun. 165, 97 (2005); J. Harting, J. Chin, M. Venturoli, and P. V. Coveney, Philos. Trans. R. Soc. London, Ser. A 363, 1895 (2005).

[24] J. Harting, M. Venturoli, and P. V. Coveney, Philos. Trans. R. Soc. London, Ser. A 362, 1703 (2004).

[25] For an explanation of volume rendering, see, e.g., W. Schroeder, K. Martin, and B. Lorensen, The Visualization Toolkit: An Object Oriented Approach to 3D Graphics, 3rd ed. (Kitware Inc., Clifton Park, NY, 2003), URL: http:// public.kitware.com/VTK/

[26] M. Wohlgemuth, N. Yufa, J. Hoffman, and E. L. Thomas, Macromolecules 34, 6083 (2001).

[27] R. Lipowsky, Nature (London) 349, 475 (1991). 